\title{
Effects of the Growth Time and the Thickness of the Buffer Layer on the Quality of the Carbon Nanotubes
}

\author{
J. Chuen ${ }^{1,2}$ \\ ${ }^{1}$ School of Physics Science and Technology, Lingnan Normal University, Zhanjiang 524048, China \\ ${ }^{2}$ School of Physics and Information Science, Hunan Normal University, Changsha 410081, China \\ Correspondence should be addressed to J. Chuen; jchuen0224@icloud.com
}

Received 29 June 2017; Accepted 28 September 2017; Published 7 November 2017

Academic Editor: Raffaele Barretta

Copyright (C) 2017 J. Chuen. This is an open access article distributed under the Creative Commons Attribution License, which permits unrestricted use, distribution, and reproduction in any medium, provided the original work is properly cited.

Direct growth of carbon nanotubes (CNTs) array onto silicon substrate by the chemical vapor deposition (CVD) is reported. Experimental results show that the thickness of the buffer layer has a significant effect on the morphology and defects of the array, and when the buffer layer is about $15 \mathrm{~nm}$, the best array on the silicon substrate can be obtained. Moreover, when the growth time is less than the threshold time (70 minutes), the array height will increase with the increase of the time. Importantly, when the growth time is higher than this threshold time, the growth of array will stop, but when the growth is continuing, the amorphous carbon and carbon can cluster, which will affect the structure of the array. These results provide a good material basis for the device, thermal, and conductivity technology.

\section{Introduction}

Carbon nanotubes (CNTs) had been discovered by Iijima since 1991 [1]; this particular one-dimensional graphite structure has attracted great research interest in experiment and theory due to its excellent electric, thermal, and mechanical properties [2, 3]. Results of these studies show that CNTs have high aspect ratio, high mechanical strength, chemical stability, and peculiar physical properties [4]. Therefore, CNTs are currently considered very prominent materials in the field of nanotechnologies. So, how to assemble CNTs into nanodevices with certain structure and function and how to make full use of the excellent performance of CNTs have become a concern. CNTs have also been applied in many situations, for example, the cold cathode electron source emitter [5] and the energy storage device electrode [6]. Thereinto, the field emission devices based on CNTs display excellent emission performance $[7,8]$. In most early studies, CNTs were mostly grown on silicon substrates [9]. In order to obtain good performance, such as device effects and electrical conductivity, direct growth on metal substrates had also been reported [10-12], in which the highly oriented CNTs array was grown by microwave plasma chemical vapor deposition (MPCVD) or plasma enhanced chemical vapor deposition (PECVD) [13]; the directional growth of CNTs was guided by the electric field bias of the plasma [14], but the growth effect was inferior to that of silicon substrate. In addition, in the area of growth of long CNTs on silicon substrate, many research teams had done a lot of work to continuously improve the height and quality of CNTs array [11-17]. In this paper, first, CNTs array with higher quality are synthesized directly on the silicon substrate by using the chemical vapor deposition (CVD) tube furnace. Secondly, we will further study the effect of the two parameters on the properties of CNTs array by adjusting the growth time and the thickness of the buffer layer. Experimental results show that the thickness of the buffer layer has a significant effect on the morphology and defects of the array, and when the buffer layer is about $15 \mathrm{~nm}$, the best array can be grown on the silicon substrate. Moreover, we have studied the effect of the growth time on the height and quality of CNTs array. Results indicate that the diameter of CNTs is more uniform when the growth time was $70 \mathrm{~min}$. These results provide a good material basis for the device, thermal, and conductivity technology. 


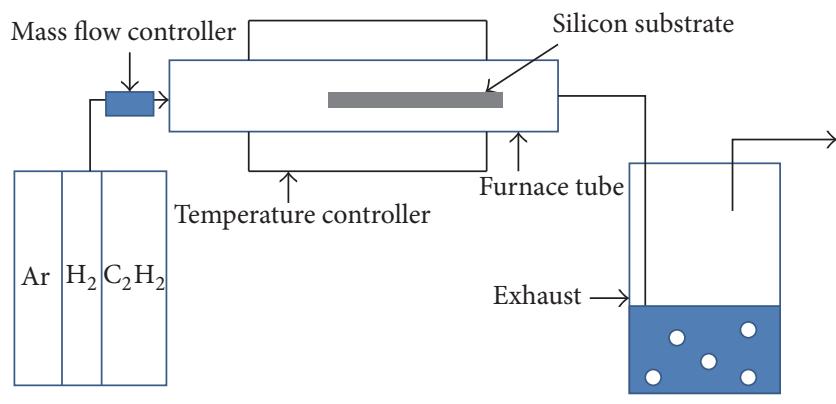

FIGURE 1: Schematic diagram of CVD apparatus.

\section{Experiment Details}

In the experiment, silicon wafer was used as the substrate for CNTs growth. Prior to growth of CNTs, the wafer needs pretreatment. This process includes cleaning, annealing, and coating. Firstly, ultrasonic cleaning is done in acetone for $5 \mathrm{~min}$ to remove organic impurities, rinse the wafer with deionized water, and it is dehydrated with anhydrous ethanol. Secondly, the buffer layer is plated. Before the catalyst is plated, we first apply the atomic layer deposition (ALD) to deposit $\mathrm{Al}_{2} \mathrm{O}_{3}$ layer with thicknesses $2 \mathrm{~nm}, 5 \mathrm{~nm}, 15 \mathrm{~nm}$, and $25 \mathrm{~nm}$. Obviously, the buffer layer is very important and plays an important role in the formation of the longer CNTs; this is because (1) $\mathrm{Al}_{2} \mathrm{O}_{3}$ has a certain surface roughness which can provide suitable conditions for the formation of uniform size and distribution of the catalyst and (2) due to the thermal stability and a huge surface area of $\mathrm{Al}_{2} \mathrm{O}_{3}$, the buffer layer can effectively organize the coagulation of catalyst particles at high temperatures. Finally, a layer of $2 \mathrm{~nm}$ Fe catalyst is plated on the buffer layer by using electron beam evaporation (EBE).

As shown in Figure 1, the complete set of equipment includes gas, mass flow meter, and temperature controller. In order to obtain CNTs array, we need air supplied with acetylene $\left(\mathrm{C}_{2} \mathrm{H}_{2}\right)$, hydrogen $\left(\mathrm{H}_{2}\right)$, and argon $(\mathrm{Ar}) . \mathrm{C}_{2} \mathrm{H}_{2}$ is the source of carbon in CNTs; $\mathrm{H}_{2}$ is reducing gas to prevent the catalyst to be oxidized. Ar in the closed furnace tube is the protective gas to prevent $\mathrm{H}_{2}$ from mixing with the air. In our experiment, firstly, we place the silicon on the quartz sheet. Secondly, we set it in the center of the furnace tube and seal the furnace tube. Thirdly, $500 \mathrm{sccm}$ of Ar is passed into the tube for 5 minutes. When the temperature reaches $500^{\circ} \mathrm{C}$, $400 \mathrm{sccm}$ of hydrogen is introduced into the furnace tube to prevent the catalyst from being oxidized. Finally, if the growth temperature reaches $750^{\circ} \mathrm{C}, 25 \mathrm{sccm}$ of Ar is introduced. At the end of the growth, the silicon substrate is coated with a layer of black material.

\section{Results and Discussion}

3.1. Effect of Buffer Layer Thickness on CNTs Array. Materials are as follows: growth atmosphere $(\mathrm{H} 2: 400 \mathrm{sccm}$; Ar: 500 sccm; C2H2: 150 sccm); Fe catalyst with thickness $2 \mathrm{~nm}$ and certain parameters of buffer layer. Growth temperature and growth time are $750^{\circ} \mathrm{C} 25$ minutes, respectively. The effect of catalyst morphology and CNTs array is studied by changing the thickness of the buffer layer.

From the results of Figure 2(a), where the thickness of the buffer layer is $2 \mathrm{~nm}$, one can see that CNTs array cannot be formed when the buffer layer is too thin. When the buffer layer is too thin, it is easy to be broken into the gap at high temperatures, and the iron catalyst particles can pass through the gap of the buffer layer to the silicon substrate and form silicide; thus, it is impossible to provide a nucleation point for the formation of CNTs arrays. In Figures 2(a)-2(d), we present the buffer layer growth effect SEM images with the thickness of $2 \mathrm{~nm}, 5 \mathrm{~nm}, 15 \mathrm{~nm}$, and $25 \mathrm{~nm}$, respectively. One can find that the buffer layer with $5 \mathrm{~nm}$ has been able to support the integrity of the catalyst to form a dense carbon tube morphology, as shown in Figure 2(b), but it does not meet the requirements. Moreover, the arrangement of the array is better when the buffer layer thickness was $15 \mathrm{~nm}$. But when the buffer layer is too thick, it will be dissolved for agglomeration phenomenon in the high temperature, as shown in Figure 2(d). It is clear that the buffer layer on the substrate forms gully morphology and shows a jumble of carbon column, so it is not the kind of tube that we need.

In Figure 3, Raman spectra are used to characterize the results of the grown arrays, Figures 2(b) and 2(c). In the Raman spectra of multiwalled CNTs arrays, there are two characteristic peaks with peak values $1580 \mathrm{~cm}^{-1}$ and $1350 \mathrm{~cm}^{-1}$, namely, the G-peak and D-peak, which correspond to the reactions of the order degree and disorder degree, respectively. In our experiment, the D-peak and Gpeak of the two samples are measured near the characteristic peaks, so it indicates that the data are reliable. Usually, the objective ID/IG ratio is used to indicate the degree of disorder and the degree of defect concentration; therefore, when the buffer layer is $15 \mathrm{~nm}, \mathrm{ID} / \mathrm{IG}=0.65$; in other words, CNTs array has a better order, a high purity, and low defect. But when the buffer layer is $5 \mathrm{~nm}, \mathrm{ID} / \mathrm{IG}=1.03$; obviously, there are more defects; the disorder is more serious. These results accord well with SEM. We can conclude that the thickness of the buffer layer has a significant effect on the morphology and defects of the array, and when the buffer layer is about $15 \mathrm{~nm}$, the best array can be grown on the silicon substrate.

3.2. Effect of the Growth Time on the Array. Keeping the growth conditions unchanged and just changing the time, we plot the array height as the function of the time, as shown in Figure 4. From the profile, it can be seen that the array height increases till threshold time (70 $\mathrm{min})$.

To illustrate this point even further, two samples with different growth time are compared. One is the sample that had just reached the maximum height (300 um) of $70 \mathrm{~min}$; the other also had reached the same maximum height, but also continued to grow 20 min. Figures 5(a)-5(d) show the characterization by SEM for different multiples. Figure 5(a) shows that a layer of CNTs vertically grows on the silicon substrate; the overall growth of the array is neat and arranged closely; also, there are many clusters on the top of the array. Figure 5(b) is schematic diagram of the middle of the array. From the result, we find that CNTs are bent upwards 


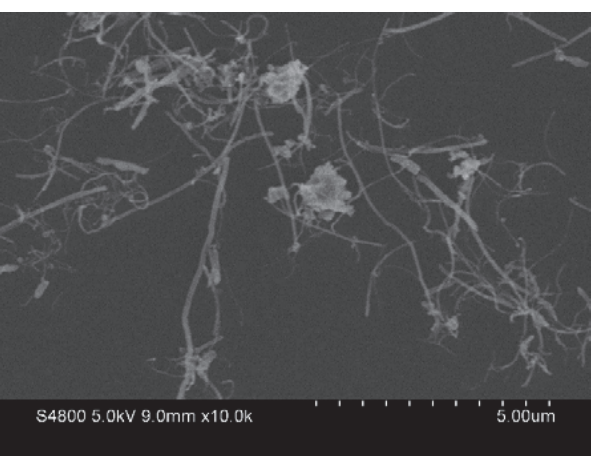

(a)

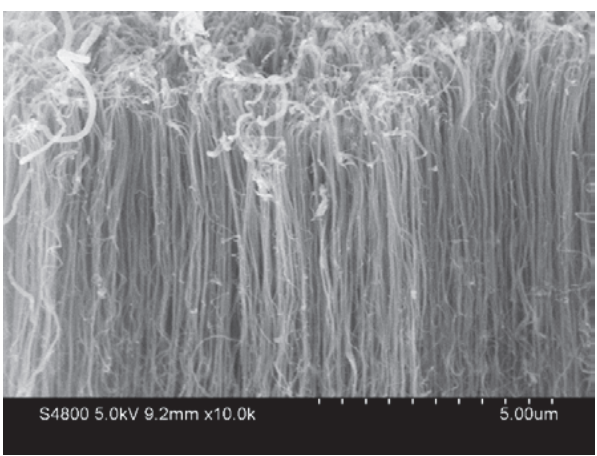

(c)

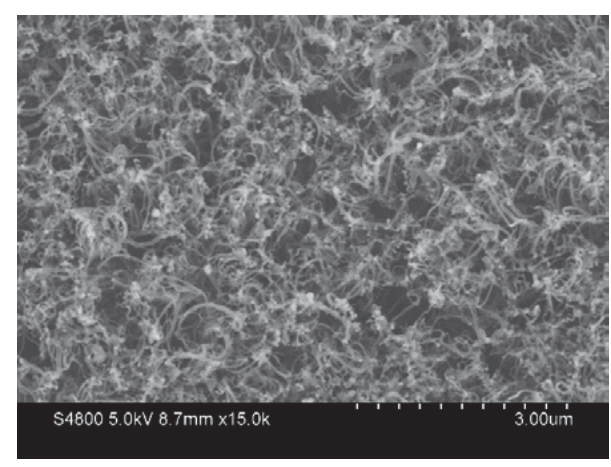

(b)

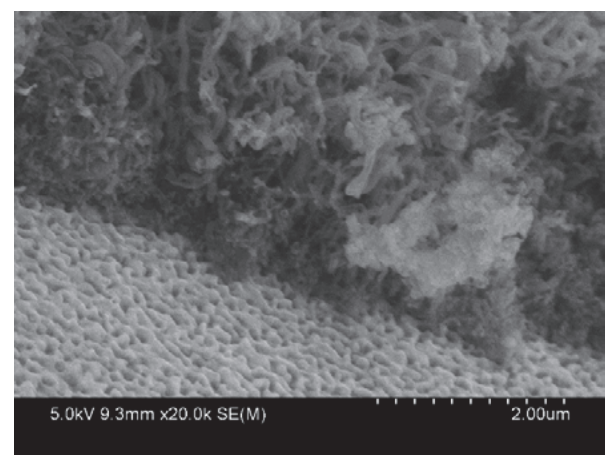

(d)

FIGURE 2: (a) $2 \mathrm{~nm}$ buffer layer growth effect SEM image, (b) $5 \mathrm{~nm}$ buffer layer growth effect SEM image, (c) $15 \mathrm{~nm}$ buffer layer growth effect SEM image, (d) $25 \mathrm{~nm}$ buffer layer growth effect SEM image.

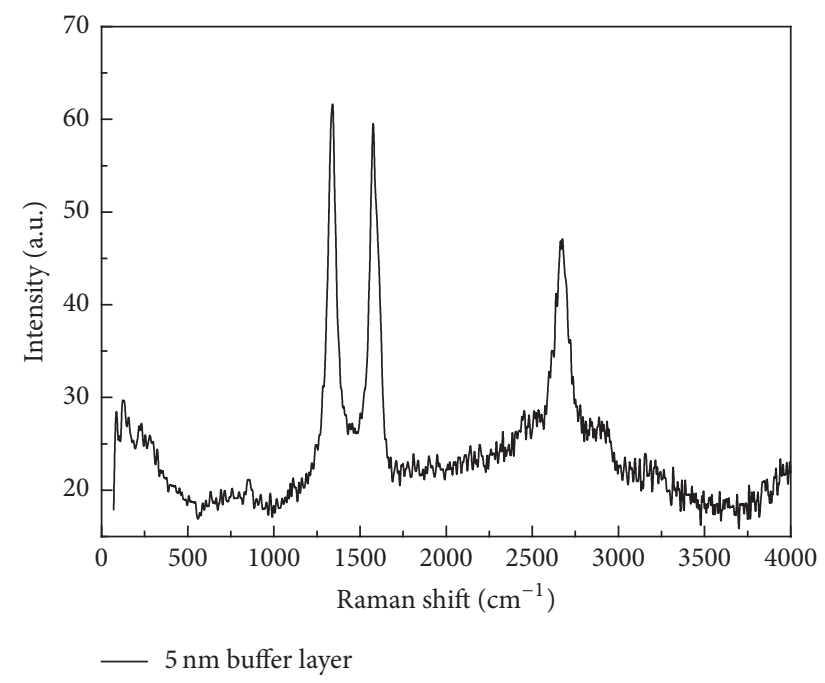

(a)

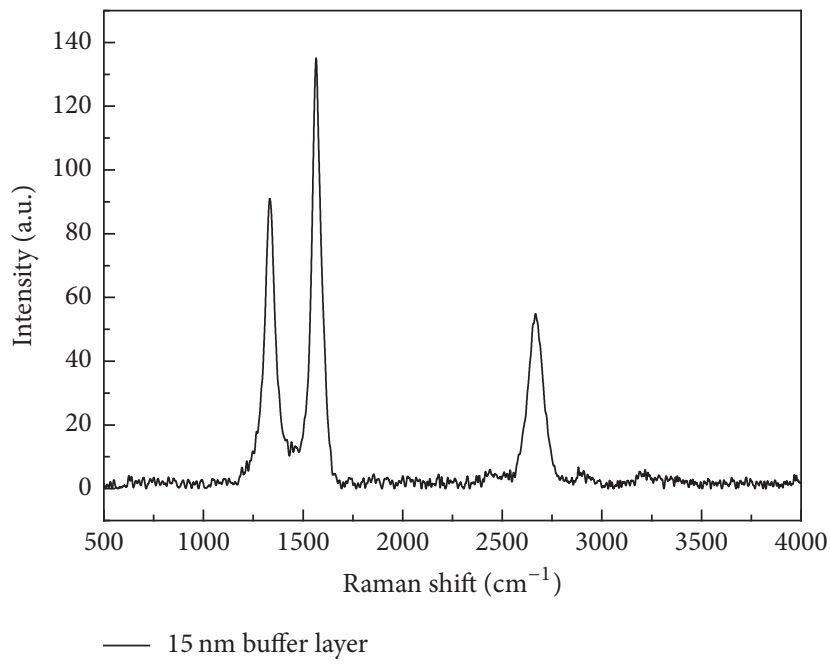

(b)

FIgURE 3: ((a) and (b)) Raman spectra of aligned CNTs.

perpendicular to the direction of the substrate, and there is no excessive cluster. Using the SEM in the three hundred thousand and five hundred thousand times to observe the top cluster structure, CNTs arrays can be clearly seen to be intertwined with each other and clearly fracture and bend, where they are mixed with amorphous carbon and catalyst particles. The carbon tube in the cluster is not as ordered as the middle of the array, and there are various forms of curvature and thickness.

In Figure 6, we present the SEM images of the sample, which has just reached the maximum height and does not continue to grow. Figure $6(\mathrm{a})$ is the top of sample and 


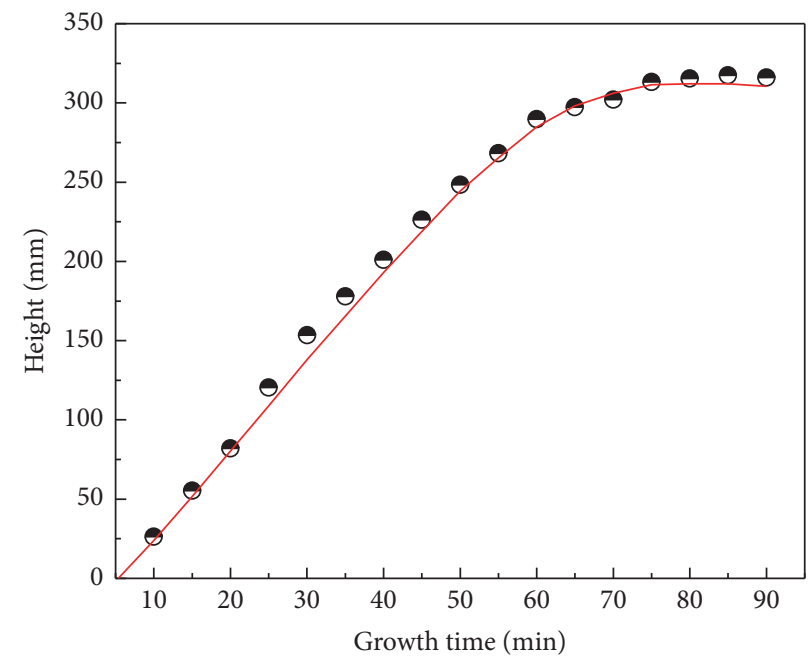

FIGURE 4: Effect of the growth time on the height of the CNT; real line is fitting result.

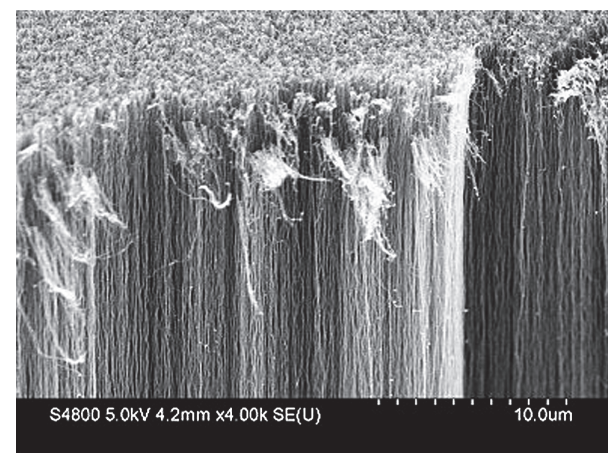

(a)

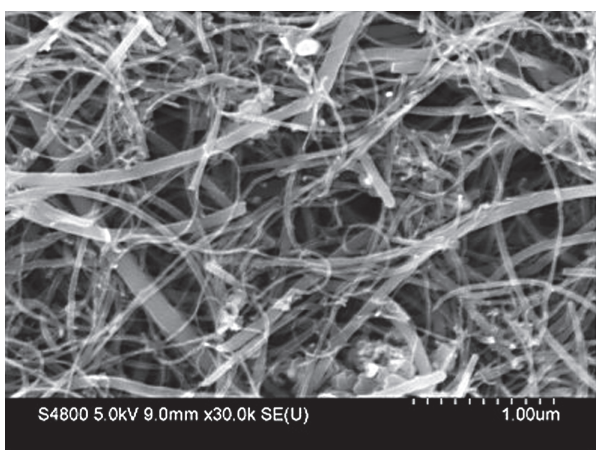

(c)

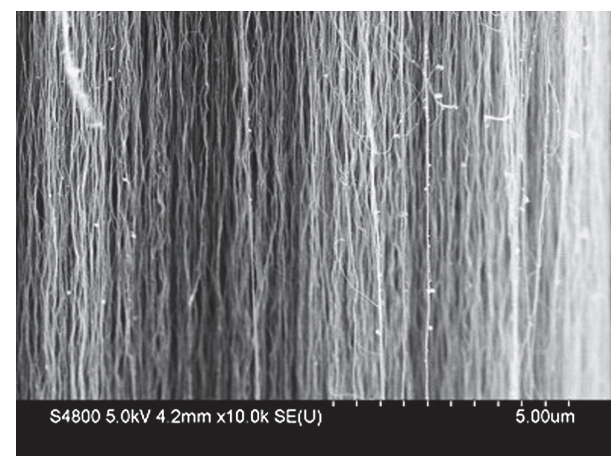

(b)

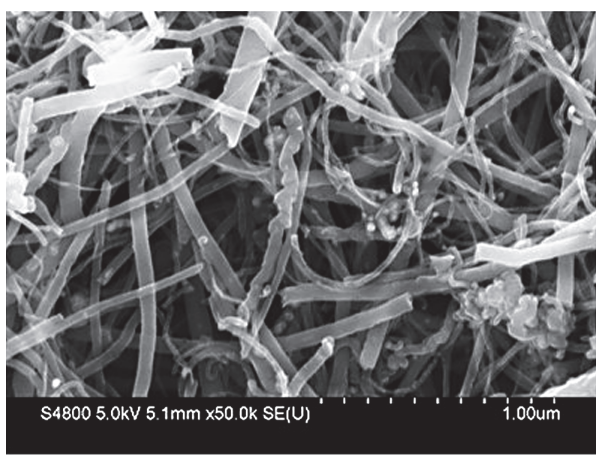

(d)

FIGURE 5: (a) 4K times the top of the array SEM image, (b) 10K times the middle of the array SEM image, (c) $30 \mathrm{~K}$ times the array cluster SEM image, and (d) $50 \mathrm{~K}$ times the array cluster SEM image.

Figure 6(b) is the SEM image of high times. It can be seen that there is no clutter cluster at the top of the array, and CNTs are arranged in an order, in which the diameter of CNTs is basically uniform, and the average diameter is about $56.4 \mathrm{~nm}$.

From the above experimental results, if the CNTS array continues to grow after the termination of the array growth, there will be random, irregular clusters and amorphous carbon. This causes serious defects at the top of the array, thus affecting the nature of the material used. We can control the array height by adjusting the growth time and maintain the purity and shape of the array while the array height is maximized. 


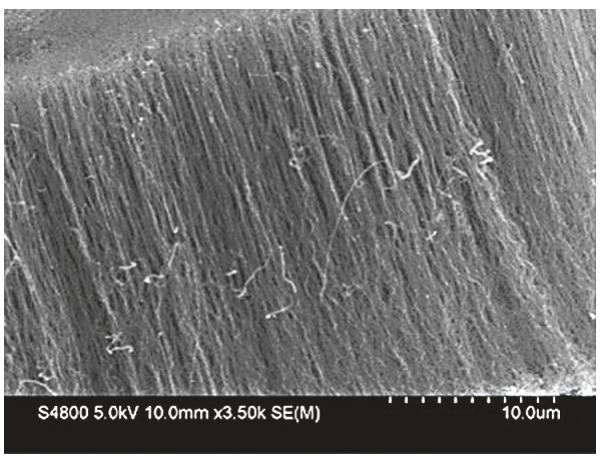

(a)

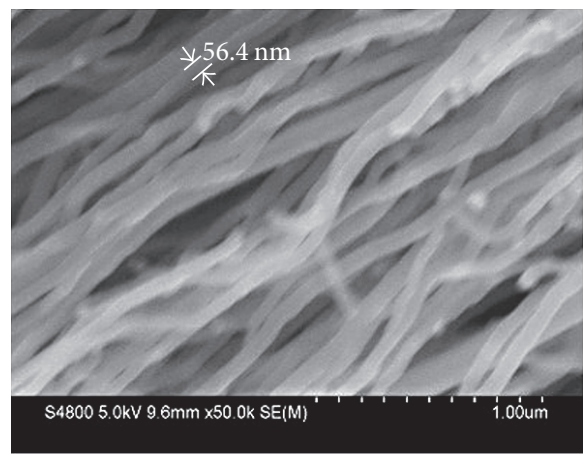

(b)

FIGURE 6: (a) 3.5K times the top of the array SEM image, (b) 50K times the top of the array SEM image.

\section{Conclusion}

Carbon nanotubes could be synthesized from acetylene and hydrogen gas directly on silicon substrates by CVD. Experimental results show that the thickness of the buffer layer has a significant effect on the morphology and defects of the array, and when the buffer layer is about $15 \mathrm{~nm}$, the best array can be grown on the silicon substrate. The results were confirmed by SEM and Raman. Moreover, when the growth time is less than 70 minutes, the array height will increase with the increase of the time. Importantly, when the growth time is higher than this time threshold, the array will not grow again, but when the arrays terminate growth, continued growth will lead to some amorphous carbon and carbon clusters, which will affect the structure of the array. From the SEM images of two samples with different growth time, we conclude that CNTs are arranged in an order; the diameter of CNTs is basically uniform when the growth time is $70 \mathrm{~min}$. If the growth time is extended to $90 \mathrm{~min}$, there will be random, irregular clusters and amorphous carbon. So, in order to obtain a better array under certain conditions of the atmosphere and the temperature, we should immediately stop after CNTs array reached the maximum height.

\section{Conflicts of Interest}

The authors declare that they have no conflicts of interest.

\section{Acknowledgments}

The work is supported by the National Natural Science Foundation of China (Grants nos. 11304276 and 11647156), the Natural Science Foundation of Guangdong Province, China (Grants nos. 2014A030307035 and 2017A030313022), the Cultivation of Innovative Talents in Colleges and Universities of Guangdong (Grant no. LYM10098), and the Natural Science Foundation of Lingnan Normal University (Grant no. ZL1004).

\section{References}

[1] S. Iijima, "Helical microtubules of graphitic carbon," Nature, vol. 354, no. 6348, pp. 56-58, 1991.
[2] H. Abuhimd, G. M. Uddin, A. Zeid, Y. J. Jung, and S. Kamarthi, "Chemical vapor deposition-grown vertically aligned singlewalled carbon nanotubes length assurance," The International Journal of Advanced Manufacturing Technology, vol. 64, no. 1-4, pp. 545-553, 2013.

[3] X. Wang, Q. Jiang, W. Xu, W. Cai, Y. Inoue, and Y. Zhu, "Effect of carbon nanotube length on thermal, electrical and mechanical properties of CNT/bismaleimide composites," Carbon, vol. 53, pp. 145-152, 2013.

[4] S. M. Dresselhaus and M. Endo, Relation of Carbon Nanotubes to Other Carbon Materials, Carbon Nanotubes, 2000.

[5] W. A. de Heer, A. Châtelain, and D. Ugarte, "A carbon nanotube field-emission electron source," Science, vol. 270, no. 5239, pp. 1179-1180, 1995.

[6] N. De Jonge, Y. Lamy, K. Schoots, and T. H. Oosterkamp, "High brightness electron beam from a multi-walled carbon nanotube," Nature, vol. 420, no. 6914, pp. 393-395, 2002.

[7] D. Park, Y. H. Kim, and J. K. Lee, "Synthesis of carbon nanotubes on metallic substrates by a sequential combination of PECVD and thermal CVD," Carbon, vol. 41, no. 5, pp. 1025-1029, 2003.

[8] M. Croci, I. Arfaoui, T. Stöckli, A. Chatelain, and J.-M. Bonard, "A fully sealed luminescent tube based on carbon nanotube field emission," Microelectronics Journal, vol. 35, no. 4, pp. 329-336, 2004.

[9] K. Hata, D. N. Futaba, K. Mizuno, T. Namai, M. Yumura, and S. Iijima, "Water-assisted highly efficient synthesis of impurityfree single-walled carbon nanotubes," Science, vol. 306, no. 5700, pp. 1362-1364, 2004.

[10] G. Y. Liu, D. Y. Zhong, S. H. Xia et al., "CNTs grown on the surface of various materials by large volume MP-CVD for VME applications," Applied Surface Science, vol. 215, no. 1-4, pp. 209213, 2003.

[11] M. Mauger, V. T. Binh, A. Levesque, and D. Guillot, "Freestanding vertically aligned arrays of individual carbon nanotubes on metallic substrates for field emission cathodes," Applied Physics Letters, vol. 85, no. 2, pp. 305-307, 2004.

[12] C.-L. Lin, C.-F. Chen, and S.-C. Shi, "Field emission properties of aligned carbon nanotubes grown on stainless steel using $\mathrm{CH} 4 / \mathrm{CO}_{2}$ reactant gas," Diamond and Related Materials, vol. 13 , no. 4-8, pp. 1026-1031, 2004.

[13] A. Kumar, S. Husain, S. Parveen et al., "Raman Characteristics of Vertically Aligned Single Wall Carbon Nanotubes Grown by Plasma Enhanced Chemical Vapor Deposition System," in Physics of Semiconductor Devices, Environmental Science and 
Engineering, pp. 563-564, Springer International Publishing, Cham, 2014.

[14] A. Mashayekhi, S. M. Hosseini, M. Hassanpour Amiri, N. Namdar, and Z. Sanaee, "Plasma-assisted nitrogen doping of VACNTs for efficiently enhancing the supercapacitor performance," Journal of Nanoparticle Research, vol. 18, no. 6, article no. 154, 2016.

[15] Z. W. Pan, S. S. Xie, B. H. Chang et al., "Very long carbon nanotubes," Nature, vol. 394, no. 6694, pp. 631-632, 1998.

[16] K. Hata and S. Iijima, "Water-assisted highly efficient synthesis of impurity-free single-walled carbon nanotubes," Science, vol. 306, no. 5700, pp. 1362-1364, 2004.

[17] S. Mao, G. Lu, and J. Chen, "Coating carbon nanotubes with colloidal nanocrystals by combining an electrospray technique with directed assembly using an electrostatic field," Nanotechnology, vol. 19, no. 45, Article ID 455610, 2008. 

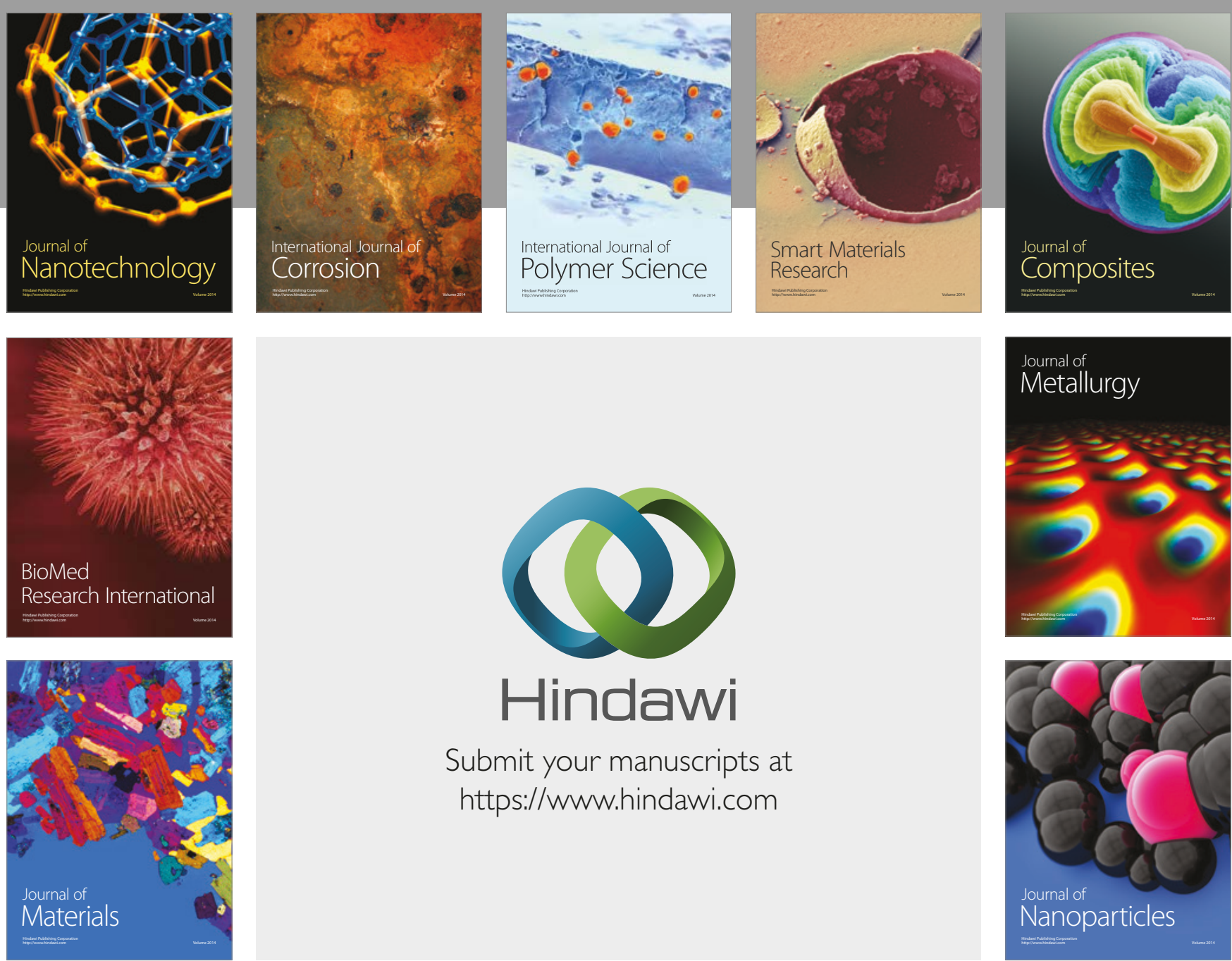

\section{Hindawi}

Submit your manuscripts at

https://www.hindawi.com
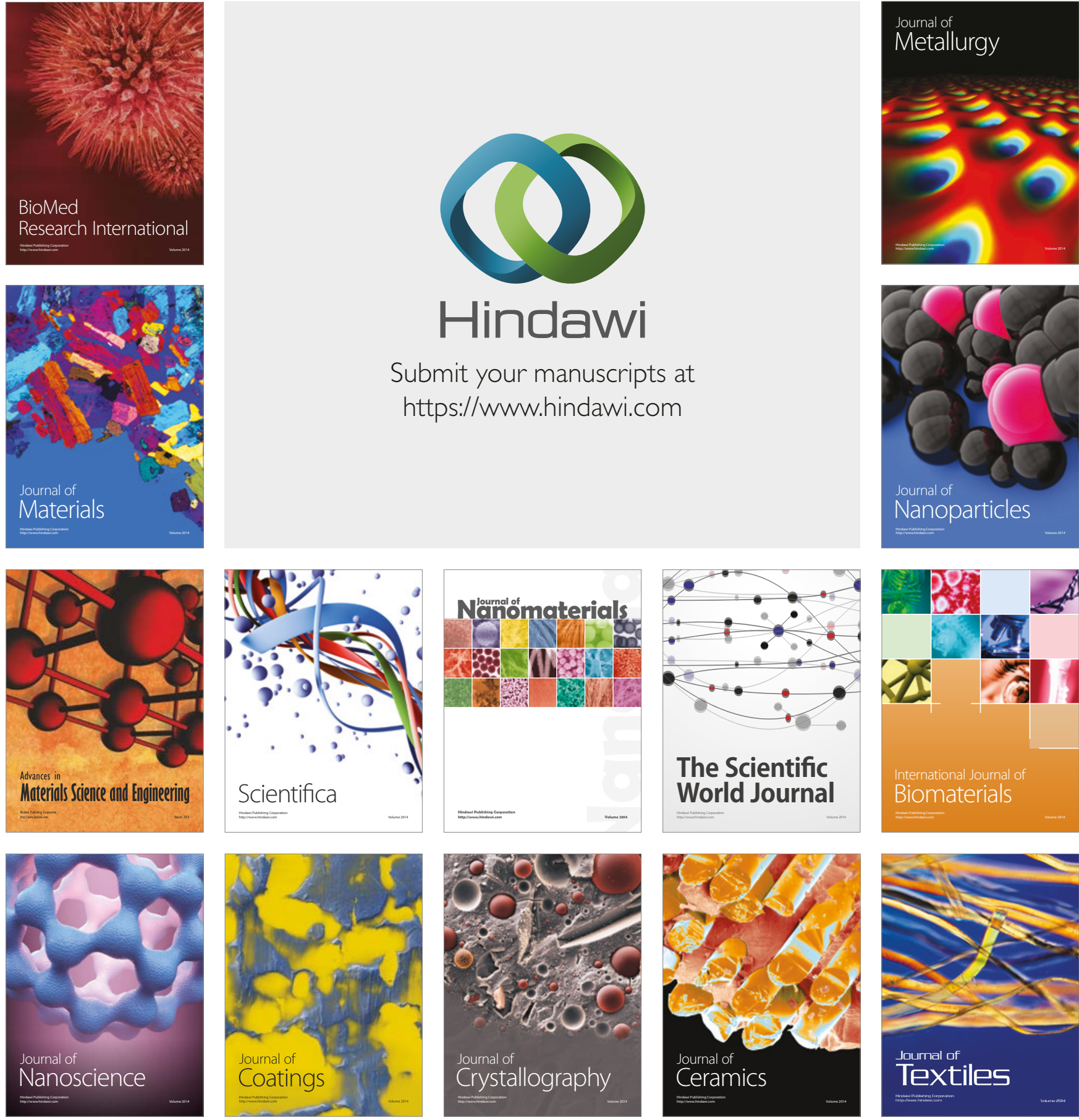

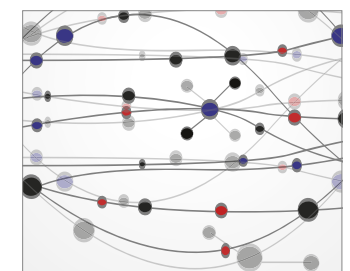

The Scientific World Journal
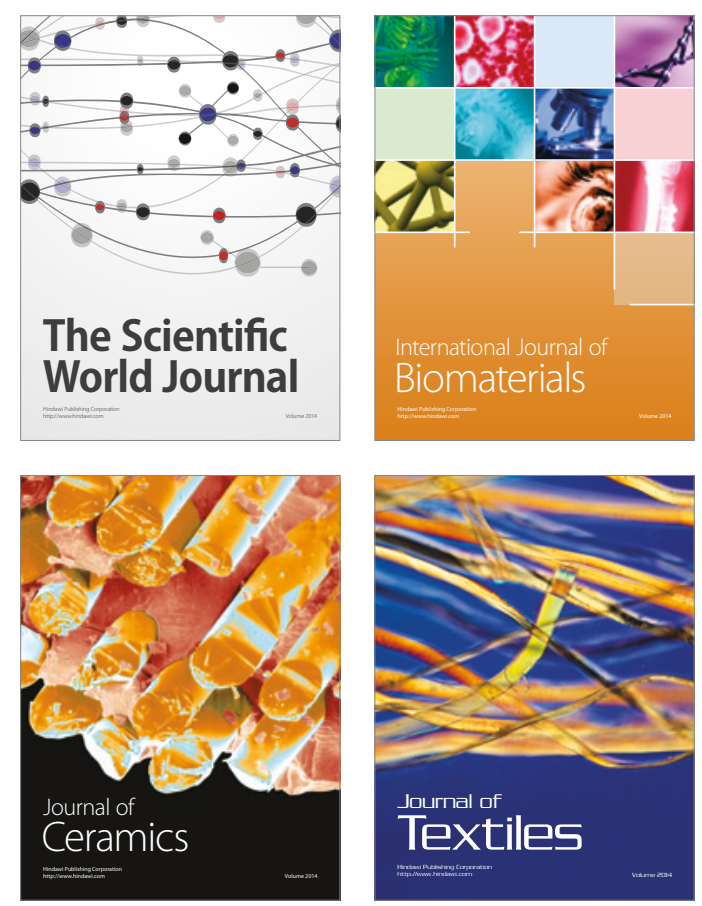